\title{
The Potassium Chloride Cotransporter KCC-2 Coordinates Development of Inhibitory Neurotransmission and Synapse Structure in Caenorhabditis elegans
}

\author{
Jessica E. Tanis, ${ }^{1,2}$ Andrew Bellemer, ${ }^{1}$ James J. Moresco, ${ }^{1}$ Biff Forbush, ${ }^{2}$ and Michael R. Koelle ${ }^{1}$ \\ Departments of ${ }^{1}$ Molecular Biophysics and Biochemistry and ${ }^{2}$ Cellular and Molecular Physiology, Yale University School of Medicine, New Haven, \\ Connecticut 06520
}

Chloride influx through GABA-gated chloride channels, the primary mechanism by which neural activity is inhibited in the adult mammalian brain, depends on chloride gradients established by the potassium chloride cotransporter KCC2. We used a genetic screen to identify genes important for inhibition of the hermaphrodite-specific motor neurons (HSNs) that stimulate Caenorhabditis elegans egg-laying behavior and discovered mutations in a potassium chloride cotransporter, $k c c-2$. Functional analysis indicates that, like mammalian KCCs, C. elegans KCC-2 transports chloride, is activated by hypotonic conditions, and is inhibited by the loop diuretic furosemide. KCC-2 appears to establish chloride gradients required for the inhibitory effects of GABA-gated and serotonin-gated chloride channels on C. elegans behavior. In the absence of KCC-2, chloride gradients appear to be altered in neurons and muscles such that normally inhibitory signals become excitatory. $k c c-2$ is transcriptionally upregulated in the HSN neurons during synapse development. Loss of KCC-2 produces a decrease in the synaptic vesicle population within mature HSN synapses, which apparently compensates for a lack of HSN inhibition, resulting in normal egg-laying behavior. Thus, KCC-2 coordinates the development of inhibitory neurotransmission with synapse maturation to produce mature neural circuits with appropriate activity levels.

\section{Introduction}

GABA is the principal inhibitory neurotransmitter in the adult mammalian brain (Mody et al., 1994). Activation of $\mathrm{GABA}_{\mathrm{A}}$ chloride channels results in chloride influx, hyperpolarizing and inactivating neurons (Farrant and Kaila, 2007). The potassium chloride cotransporter KCC2, one of four KCCs in vertebrates (Gillen et al., 1996; Payne et al., 1996; Mount et al., 1999), uses the neuronal potassium gradient to drive potassium and chloride efflux, establishing the chloride concentration gradient necessary for the hyperpolarizing inhibitory effect of GABA in mature neurons. Because immature neurons fail to express KCC2 but do express the sodium potassium chloride cotransporter NKCC1, which drives chloride influx, intracellular chloride levels remain high, and activation of $\mathrm{GABA}_{\mathrm{A}}$ channels results in an excitatory rather than inhibitory response (Rivera et al., 1999; Ben-Ari, 2002).

The excitatory action of GABA appears to cause activitydependent synaptic changes during early development that are nec-

\footnotetext{
Received April 27, 2009; revised June 24, 2009; accepted July 1, 2009.

This work was supported by National Institutes of Health (NIH) Grants NS036918 (M.R.K.) and GM083340 (B.F.), a National Science Foundation graduate fellowship and NIH Training Grant DK007259 (J.E.T.), and NIH Fellowship NS060432 (A.B.). We thank the Caenorhabditis Genetics Center for strains, Kwang Kim, Susumu Tomita, and Walter Boron for Xenopus oocytes, and Keith Nehrke for sharing unpublished data about C. elegans transporters.

Correspondence should be addressed to Dr. Michael R. Koelle, Yale University School of Medicine, Department of Molecular Biophysics and Biochemistry, 333 Cedar Street, SHM CE30, New Haven, CT 06520-8024. E-mail: michael.koelle@yale.edu.

J. J. Moresco's present address: Department of Chemical Physiology, The Scripps Research Institute, La Jolla, CA 92037.

DOI:10.1523/JNEUROSCI.1989-09.2009

Copyright $\odot 2009$ Society for Neuroscience $\quad$ 0270-6474/09/299943-12\$15.00/0
}

essary to produce mature neural circuits (Owens and Kriegstein, 2002). Premature expression of KCC2, which results in an early shift to inhibitory GABA signaling, results in abnormal neuron morphology and synapse formation (Chudotvorova et al., 2005; Akerman and Cline, 2006; Cancedda et al., 2007, Reynolds et al., 2008). KCC2 expression during late development is also important for synapse maturation, because neurons from KCC2 knockout mice exhibit elongated dendritic spines and reduced synapse number ( $\mathrm{Li}$ et al., 2007). Thus, KCC2 upregulation appears to control the onset of inhibitory neurotransmission and synapse maturation to produce mature neural circuits.

In addition to promoting the developmental shift in the effect of GABA from excitatory to inhibitory, changes in KCC2 expression (Rivera et al., 2004; Liu et al., 2006) or phosphorylation (Wake et al., 2007) may alter the magnitude of chloride gradients to modulate inhibitory neurotransmission. Determining how KCC2 regulates neuronal activity is essential for understanding epilepsy, because a decrease in neuronal KCC2 levels has been observed in epileptic patients (Huberfeld et al., 2007), and mice with a targeted deletion of the KCC2b isoform have spontaneous seizures that result in death (Woo et al., 2002).

Caenorhabditis elegans egg laying is a highly regulated behavior stimulated by serotonin release from the pair of hermaphroditespecific motor neurons (HSNs) (Trent et al., 1983). The HSNs are the most highly characterized neurons in C. elegans, and sophisticated tools are available for analyzing their development and function (Shen et al., 2004; Adler et al., 2006; Ding et al., 2007; Tanis et al., 2008). The putative transmembrane receptor EGL-47 is expressed on the HSN neurons and inhibits HSN activity with- 
out disrupting neuron development or morphology (Moresco and Koelle, 2004). Using a dominant (dm) activating mutant of EGL-47 as a tool to inactivate the HSNs, we performed a suppressor screen to identify genes necessary for inhibition of HSN activity and isolated mutations in the novel gene $k c c-2$, which encodes a potassium chloride cotransporter. C. elegans $k c c-2$ null mutants are viable but exhibit defects in inhibitory neurotransmission and HSN synapse size. This provided us with a unique opportunity to investigate how KCC action in an identified neuron regulates neural function, synapse morphology, and behavior.

\section{Materials and Methods}

Nematode culture. C. elegans strains were cultured at $20^{\circ} \mathrm{C}$ as described by Brenner (1974). The wild-type strain was Bristol N2; all other strains used in this work are listed in supplemental Materials and Methods (available at www.jneurosci.org as supplemental material).

egl-47(dm) suppressor screen. Synchronous populations of fourth larval (L4) stage egl-47(n1081dm) animals were mutagenized with methanesulfonic acid ethyl ester and transferred to nematode growth media (NGM) agar plates. F1 adults were treated with bleach/ $\mathrm{NaOH}$ to release their eggs, which were placed on NGM plates to produce plates each carrying $\sim 200$ synchronized F2 animals. Eighteen hours after the F2 animals reached the late L4 stage, the plates were screened and F3 eggs that had been laid were picked. We screened 109,000 mutagenized genomes and identified 28 independent mutants, seven of which formed a complementation group that was mapped to the $k c c-2$ gene as described in supplemental Materials and Methods (available at www.jneurosci.org as supplemental material).

Functional expression in Xenopus oocytes. The C. elegans $k c c-2 b$ cDNA was inserted into the Poll oocyte expression vector (Caron et al., 2000) to generate plasmid pJT93A. cRNA was synthesized with T7 RNA polymerase (Ambion) and injected into defolliculated stage IV-VI Xenopus laevis oocytes ( $20.5 \mathrm{ng}$ of cRNA per oocyte). Oocytes were incubated $3 \mathrm{~d}$ at $17^{\circ} \mathrm{C}$ in ND96 medium.

Transporter activity was determined by measuring radioactive ${ }^{86} \mathrm{Rb}$ influx into individual oocytes using an automated 96-well plate machine (Darman and Forbush, 2002). Oocytes were preincubated for $30 \mathrm{~min}$, followed by a $30 \mathrm{~min}{ }^{86} \mathrm{Rb}$ flux period. Hypotonic media contained the following (in mM): $73 \mathrm{NaCl}, 2 \mathrm{RbCl}, 1 \mathrm{CaCl}_{2}, 1 \mathrm{MgCl}_{2}$, and 5 HEPES. Isotonic solution consisted of $50 \mathrm{~mm}$ sucrose in hypotonic media. In the hypotonic solution without chloride, gluconate and sulfate were substituted for the chloride. Oubain at $0.01 \mu \mathrm{M}$ and $5 \mu \mathrm{M}$ bumetanide were added to solutions to reduce ${ }^{86} \mathrm{Rb}$ influx from the endogenous $\mathrm{Na}^{+} / \mathrm{K}^{+}$ ATPase and NKCC, respectively. To determine sensitivity to loop diuretics, increasing concentrations of furosemide and bumetanide were added to hypotonic solution. $\mathrm{IC}_{50}$ values were obtained using a nonlinear least squares regression to fit individual experiments to the Langmuir binding equation for the inhibitors. Statistical significance was determined using Student's $t$ test on both raw and normalized data. All experiments were conducted at least three times with six or more oocytes in each condition per experiment.

Transgenes. Germ-line transformation of transgenes was performed using standard techniques (Mello et al., 1991). The genomic clones B0350, C46G7, and H16014 were injected at $20 \mathrm{ng} / \mu \mathrm{l}$ with the coinjection marker myo-2::gfp (JKL449.1; a gift from A. Fire, Stanford University, Stanford, CA) at $10 \mathrm{ng} / \mu \mathrm{l}$ into the $v s 132 ; \mathrm{egl}-47(\mathrm{n} 1081 \mathrm{dm})$ mutant, and transgenic strains were analyzed for rescue of $v s 132$. Kcc promoter:::fp::kcc $3^{\prime}$ untranslated region (UTR) constructs used to determine the expression patterns of $k c c-1$ (pJT92A), $k c c-2$ (pJT74A, pJT109A, pJT110A), and $k c c-3$ (pJT68A) were injected at $100 \mathrm{ng} / \mu \mathrm{l}$ with the lin-15 rescuing construct pL15EK (50 ng/ $\mu \mathrm{l})$ into MT8189 lin-15(n765ts). Additional analysis of the $k c c-2$ expression pattern was performed by using long-range PCR to amplify the $k c c$ promoter::gfp::kcc 3' UTR cassette from pJT109A or pJT110A and directly injecting the PCR product $(50 \mathrm{ng} / \mu \mathrm{l})$ with pL15EK (50 ng/ $\mu \mathrm{l})$ into MT8189 (Etchberger and Hobert, 2008).

Egg-laying system cell-specific expression constructs were created by inserting the $k c c-2 b c D N A$ into the HSN and egg-laying muscle (ELM) expression vectors (Tanis et al., 2008), pJT87A and pJT88A, respectively. Constructs used to express green fluorescent protein (GFP) in the HSNs
(pJM60A) and the ELMs (pBH34.21) served as controls. The HSN expression construct was injected at $100 \mathrm{ng} / \mu \mathrm{l}$ with pJKL449.1 $(10 \mathrm{ng} / \mu \mathrm{l})$ into LX1021 kcc-2(vs132); egl-47(n1081). The ELM expression construct was injected at $10 \mathrm{ng} / \mu \mathrm{l}$ with pJKL449.1 $(10 \mathrm{ng} / \mu \mathrm{l})$ into LX1021. Additional cell-specific rescue constructs were made with a myo-3 promoter, which was used to drive cell-specific expression of the $k c c-2 b c D N A$ (pAB29) and unc-49 cDNA (pAB32) in the body-wall muscles. pAB25, which was used to express GFP in the body-wall muscles, served as a control. Body-wall muscle expression constructs were injected at 15 $\mathrm{ng} / \mu \mathrm{l}$ with the coinjection marker pSAK2.

Behavioral assays. Animals for behavioral assays were picked as L4 larvae and grown $18 \mathrm{~h}$ (see Fig. 1 E), $24 \mathrm{~h}$ (see Fig. 5A,B,G,H and supplemental Fig. 6, available at www.jneurosci.org as supplemental material), $36 \mathrm{~h}$ (see Fig. $6 E$ and supplemental Fig. $1 A$, available at www.jneurosci.org as supplemental material), or $40 \mathrm{~h}$ (see Fig. $6 F, G$ and supplemental Fig. $1 \mathrm{~B}$, available at www.jneurosci.org as supplemental material) at $20^{\circ} \mathrm{C}$ to generate staged adults. egl-47( $\mathrm{dm}$ ) suppression was measured by counting the number of eggs laid by five hermaphrodites within $18 \mathrm{~h}$ after the L4 stage. Unlaid eggs and the percentage of early-stage eggs were quantitated as described previously (Chase and Koelle, 2004). Egg production was determined by adding the total number of eggs laid by one animal by $24 \mathrm{~h}$ after the L4 stage with the total number of eggs in utero at that point. The serotonin and muscimol response assays are described in supplemental Material and Methods (available at www.jneurosci.org as supplemental material).

Fluorescence microscopy. Transgenic animals were immobilized with 3 mM levamisole on agar pads. KCC expression pattern images were obtained with a Carl Zeiss LSM 510 confocal microscope and reconstructed using Volocity software (Improvision).

Quantitative fluorescence microscopy of animals expressing the $k c c-2 c$ promoter::gfp::kcc-23' UTR reporter transgene was performed on a Carl Zeiss Axioskop. Developmental age was scored based on distal tip cell migration (L3) and vulval morphology (L4), and staged adult animals were cultured $12 \mathrm{~h}$ after late L4. Single HSN images were taken through the center of the cell body for 30 animals per developmental stage. Mean GFP intensity for each cell body was determined using Openlab software (Improvision). Average GFP intensity at the L3 and adult stages was significantly different from average GFP intensity at the L4 stage $(p<0.0001)$.

SNB-1::GFP and Discosoma red fluorescent protein 2 (DsRed2) were expressed in the HSNs at the lowest possible expression level that still allowed for adequate imaging with a Carl Zeiss LSM 510 confocal microscope, and transgene expression did not affect egg-laying behavior (Tanis et al., 2008). Staging and immobilization of animals as well as image acquisition and analysis of the number, volume, and fluorescence intensity of SNB-1::GFP-labeled synaptic vesicle clusters were performed as described previously (Tanis et al., 2008). Total SNB-1::GFP-labeled volume was measured using four different GFP intensity cutoffs, and the difference between the wild-type and $k c c$ - 2 was significant for each analysis. DsRed 2 varicosities were defined as regions in the HSN process wider than $1 \mu \mathrm{m}$ in diameter in any single dimension within $10 \mu \mathrm{m}$ of the vulva; total varicosity volume was determined by adding the volumes of all the DsRed varicosities in a single neuron. Total varicosity fluorescence is a measure of average varicosity voxel intensity multiplied by the total number of voxels in the varicosity region for each neuron.

\section{Results}

\section{Mutations in $k c c-2$ suppress the egl-47(dm) inhibition of HSN activity}

Dominant mutations in the putative transmembrane receptor EGL-47 inhibit HSN activity to inhibit egg laying (Moresco and Koelle, 2004). Whereas wild-type adults retained $13.3 \pm 0.4$ unlaid eggs in utero, the egl-47( $\mathrm{dm})$ mutant retained $54.9 \pm 1.0$ eggs (Fig. $1 A, B$ ). Serotonin released from the HSN neurons stimulates contraction of the ELMs, resulting in egg-laying events (Trent et al., 1983). Application of exogenous serotonin, but not serotonin reuptake inhibitors, stimulates egg laying in the egl$47(\mathrm{dm})$ mutant, indicating that the ELMs are functional and that 

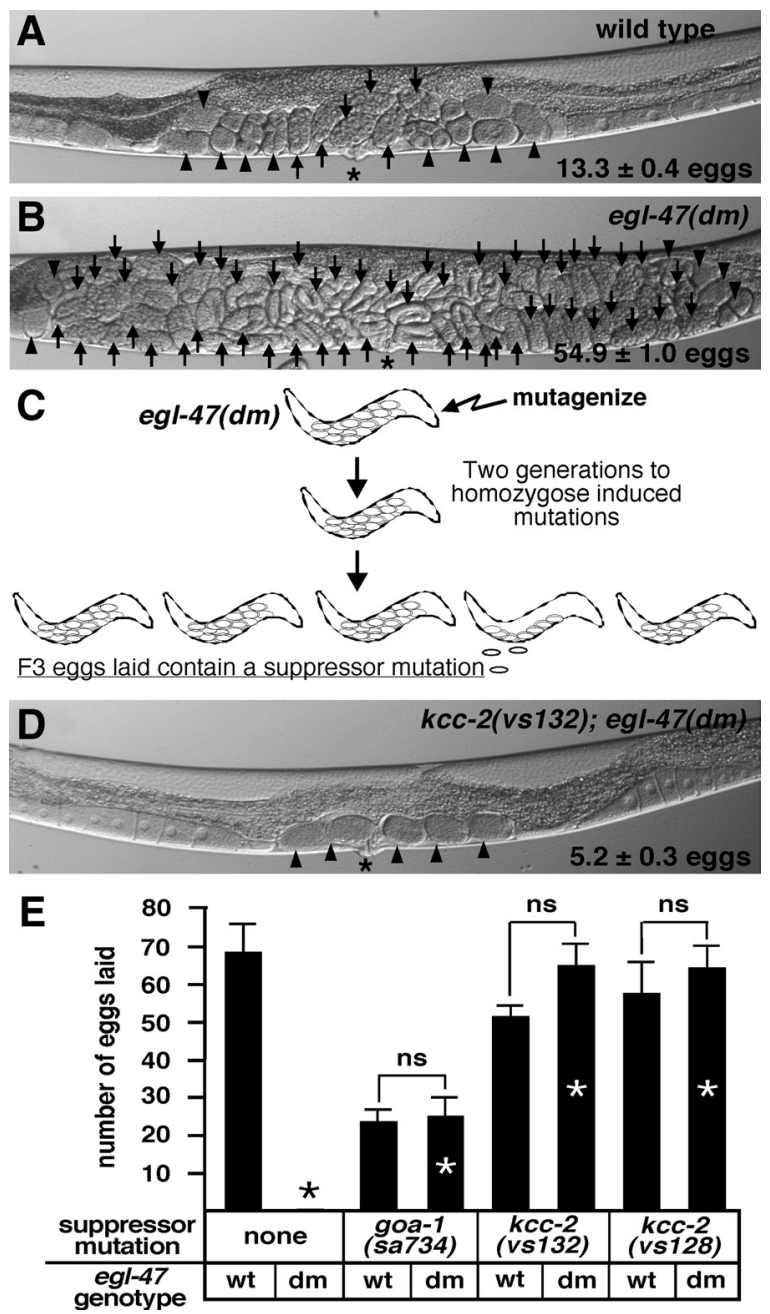

Figure 1. Mutations in kcc-2 suppress the egl-47(dm) egg-laying defect. $\boldsymbol{A}, \boldsymbol{B}$, Representative wild-type and egl-47(dm) animals, respectively. For $\boldsymbol{A}, \boldsymbol{B}$, and $\boldsymbol{D}$, the average number of unlaid eggs ( $\pm 95 \%$ confidence interval), eggs with eight cells or fewer (arrowheads), eggs with greater than eight cells (arrows), and the vulva (asterisk) are as indicated. C, A genetic screen was performed to identify suppressors of egl-47(dm). The egl-47( $\mathrm{dm})$ mutant retained many unlaid eggs in utero and never laid any eggs by $18 \mathrm{~h}$ after the late $\mathrm{L} 4 \mathrm{stage}$. egl-47(dm) animals were mutagenized, and F3 eggs laid by the $\mathrm{F} 2$ generation $18 \mathrm{~h}$ after $\mathrm{L} 4$ were picked as suppressors. D, A representative kcc-2(vs 132); egl-47(dm) animal. E, By $18 \mathrm{~h}$ after $\mathrm{L} 4$, the wild type (wt) laid many eggs, whereas the egl-47(dm) mutant never laid an egg. Mutations in goa-1 and $k c c-2$ suppressed the egl-47(dm) egg-laying defect (compare bars with white asterisks to the black asterisk denoting a measurement of zero eggs). The goa-1 mutant laid fewer eggs than the wild type because the goa-1 mutant produced fewer eggs. The number of eggs laid in $\boldsymbol{E}$ is by five animals by $18 \mathrm{~h}$ after L4; error bars show SE. "ns" over brackets indicates measurements that are not significantly different, demonstrating full suppression of $\mathrm{egl}-47(\mathrm{dm})$.

the egg-laying defect is caused by a lack of serotonin release from the HSNs (Moresco and Koelle, 2004). Because HSN development and morphology are normal in the egl-47(dm) mutant (Moresco and Koelle, 2004), the egl-47( $d m$ ) mutation apparently inhibits HSN activity.

The egl-47(dm) mutant never lays eggs as a young adult, providing a sensitive genetic background to screen for suppressor mutations that reactivate egg laying. By $18 \mathrm{~h}$ after the late L 4 stage, five wild-type animals laid $68.3 \pm 7.6$ eggs, whereas the egl$47(\mathrm{dm})$ mutant has never laid an egg in this assay (Fig. $1 \mathrm{E}$ ) (data not shown). The neural G-protein G $\alpha_{\mathrm{o}}$, known as GOA-1 in $C$. elegans, acts in the HSNs to inhibit their activity (Tanis et al., 2008). EGL-47 may act in part through GOA-1 because goa-1(lf) mutations suppress the lack of egg laying in young adult egl-
47( $\mathrm{dm}$ ) mutants (Fig. 1E) (Moresco and Koelle, 2004). More detailed epistasis experiments, however, show that EGL-47 has at least some of its effects by acting independently of GOA-1 (supplemental Fig. 1, available at www.jneurosci.org as supplemental material).

We used the genetic screen schematized in Figure $1 C$ to identify suppressor mutations that allow young adult egl$47(\mathrm{dm})$ animals to lay eggs. Mutations in genes required for GOA-1 signaling (to be described elsewhere) and genes that enable inhibitory neurotransmission in the egg-laying system were isolated. We mapped the egl-47( $\mathrm{dm}$ ) suppressor vs132 (supplemental Fig. 2, available at www.jneurosci.org as supplemental material) and found a mutation in a gene that we named $k c c-2$ based on its similarity to vertebrate KCCs. Compared with the $54.9 \pm 1.0$ eggs retained by the egl-47( $\mathrm{dm})$ mutant, the $k c c-2(v s 132)$; egl-47 $(\mathrm{dm})$ double mutant retained only $5.2 \pm 0.3$ eggs in utero (Fig. $1 B, D)$. The $k c c-2(v s 132)$; egl-47( $d m$ ) mutant laid a nearly wild-type number $(64.8 \pm 6.2)$ of eggs by $18 \mathrm{~h}$ after L4, indicating that the $k c c-2$ mutation reduced the number of unlaid eggs in the double mutant by reactivating egg laying (Fig. $1 E$ ). The $k c c$-2(vs132); egl-47( $d m$ ) double mutant and the $k c c-2$ (vs132) single mutant laid statistically indistinguishable numbers of eggs (Fig. $1 \mathrm{E}$ ), indicating that $k c c-2$ fully suppresses egl-47( $d m$ ). Seven alleles of $k c c-2$ were identified in the egl-47( $\mathrm{dm})$ suppressor screen (Fig. 2A) (supplemental Fig. 3, available at www.jneurosci.org as supplemental material). All showed similar egl-47( $\mathrm{dm})$ suppression levels, and, because one allele (vs132) causes an early stop codon and is a predicted null mutation, all alleles are likely lossof-function mutations.

\section{KCC-2 has little effect on the functional output of the HSN motor neurons in the absence of challenges to the egg-laying system}

We crossed $k c c-2$ mutations out of the egl-47( $d m)$ background and used egg-laying assays as a readout to study HSN function in $k c c-2$ single-mutant animals. Whereas the wild type retained $13.3 \pm 0.4$ unlaid eggs in utero, the $k c c-2$ mutant retained significantly fewer eggs $(10.9 \pm 0.3)$. We determined that the reduction in the number of unlaid eggs in the $k c c-2$ mutant is the result of a decrease in egg production, not an increase in egg-laying rate. In wild-type C. elegans, eggs are internally fertilized and undergo cell divisions in utero before being laid, such that only 5\% of freshly laid eggs are at an early stage, with eight cells or fewer. Mutants with an increased frequency of egg-laying behavior lay a dramatically increased percentage of early-stage eggs, making this a sensitive measure of hyperactive egg laying (Chase and Koelle, 2004). The $k c c-2$ mutant laid $10.7 \pm 5.5 \%$ early-stage eggs, which was not significantly different from the $7.7 \pm 4.4 \%$ early-stage eggs laid by the wild type, indicating that the wild-type and $k c c-2$ mutant have similar egg-laying rates. Egg production was analyzed by isolating L4 animals and, $24 \mathrm{~h}$ later, adding the total number of eggs that each animal had laid with the number of eggs remaining in utero. Whereas the wild type produced $52.6 \pm 2.7$ eggs, the $k c c-2$ mutant only produced $26.3 \pm 3.1$ eggs. Although this decrease in egg production is a significant phenotype of $k c c-2$ mutants, it is presumably unrelated to HSN function. Thus, our results suggest that the functional output of the HSN motor neurons is relatively normal in $k c c-2$ single mutants unless these animals are challenged with the egl-47( $\mathrm{dm}$ ) mutation or by treatment with the GABA agonist muscimol (see below). 

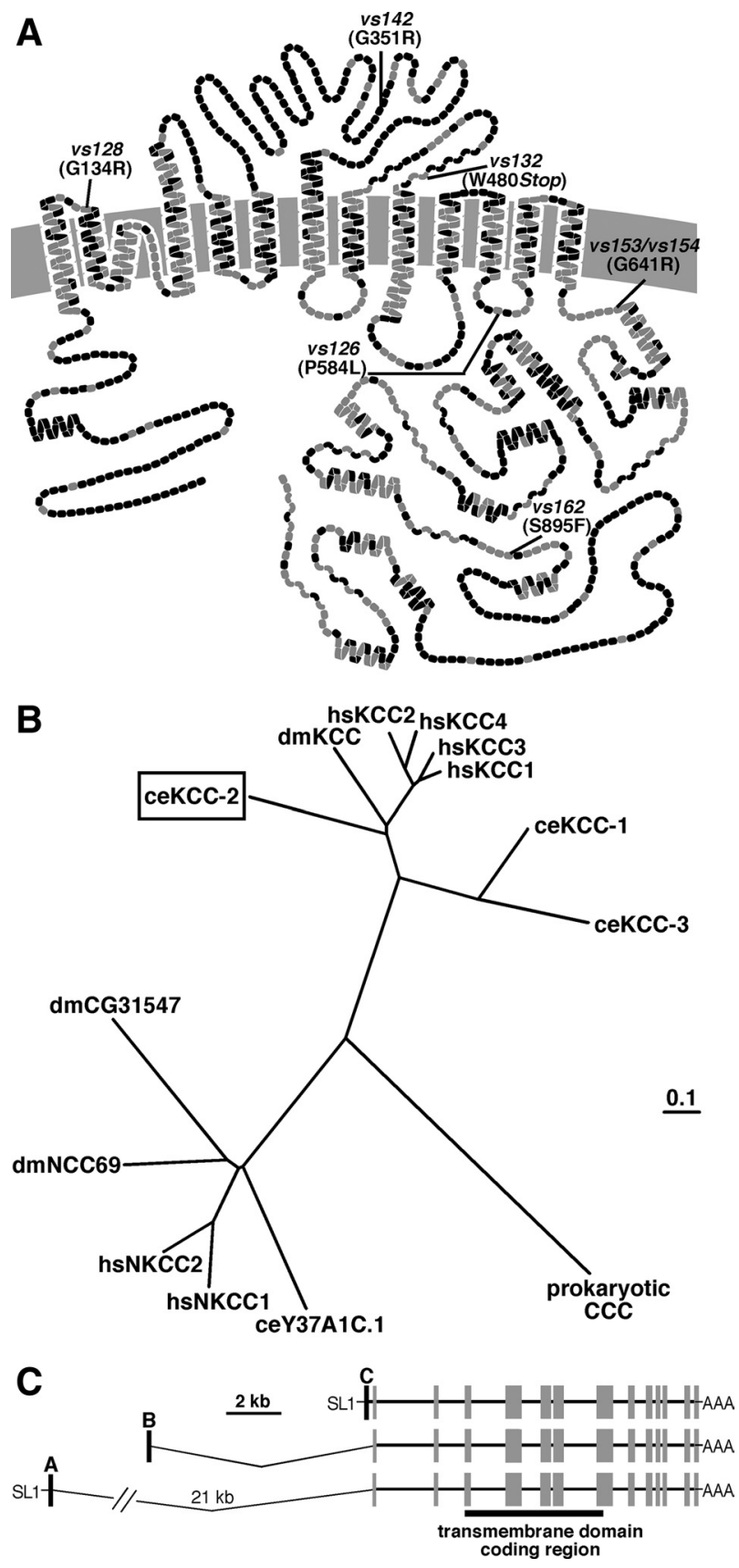

Figure 2. C. elegans KCC-2 exhibits sequence similarity to mammalian KCCS. A, Predicted transmembrane structure of $C$. elegans $\mathrm{KCC}-2$ compared with the sequence of rat $\mathrm{KCC}$. Each amino acid is represented by a circle; gray circles indicate identical amino acids, and black circles represent non-identical amino acids. The locations of the $k c c-2$ mutations are as indicated. $\boldsymbol{B}$ Phylogenetic comparison of C. elegans KCC-2 (ceKCC -2 ; boxed) to the four human KCCS (hsKCC1-hsKCC4), Drosophila melanogaster KCC (dmKCC), two additional C. elegans KCC homologs (ceKCC -1 and ceKCC -3 ), the human sodium potassium chloride cotransporters (hsNKCC1 and hsNKCC2), two predicted D. melanogaster NKCCS (dmCG31547 and dmNCC69), a predicted C. elegans NKCC (ceY37A1C.1), and a prokaryotic cation chloride cotransporter (CCC). C, Gene structure of $C$. elegans $k c c-2$. Three alternative splice forms, $k c c-2 a, k c c-2 b$, and $k c c-2 c$, each contain a different $5^{\prime}$ exon (black boxes) that splices onto common exons (gray boxes) encoding the transmembrane domains (black bar). The SL1 trans splice leader (SL1) was identified on the $A$ and $C$ isoforms but not on the $B$ isoform.

\section{C. elegans $k c c-2$ encodes a putative potassium} chloride cotransporter

C. elegans KCC-2 resembles previously characterized KCCs, containing 12 predicted transmembrane domains, large intracellular
$\mathrm{N}$ - and $\mathrm{C}$ termini, and a large extracellular loop between the fifth and sixth transmembrane domains (Fig. 2A). Based on the functional similarities between $C$. elegans KCC-2 and rat KCC2 described below, we compared the sequences of these two transporters. The highest levels of conservation are observed in the transmembrane domains and regions of the $\mathrm{C}$ terminus, whereas there is little similarity in the $\mathrm{N}$ terminus or large extracellular loop (Fig. 2A) (supplemental Fig. 3, available at www.jneurosci.org as supplemental material). $k c c-2$ mutations identified in the egl-47 $(\mathrm{dm})$ screen were primarily found in conserved residues (Fig. 2A) (supplemental Fig. 3, available at www.jneurosci.org as supplemental material) throughout the protein.

We performed a phylogenetic analysis of the evolutionary relationships between $C$. elegans KCC-2 and other cation chloride cotransporters (Fig. 2B) (supplemental Fig. 4A, available at www.jneurosci.org as supplemental material). C. elegans KCC-2 is $43-47 \%$ identical to the four human KCCs. Although there is not one clear human ortholog, KCC-2 exhibits a higher level of conservation with the four human KCCs than with KCC-1 and KCC-3, two other putative C. elegans KCCs (Fig. 2 B). Using reverse transcription-PCR, we identified three alternative splice forms of $k c c-2$, each with a different first exon that contains a translation start and splices onto common exons encoding the transmembrane domains (Fig. 2C) (supplemental Fig. 4B-E, available at www.jneurosci.org as supplemental material). Such alternative splicing also affects the $\mathrm{N}$ termini of vertebrate KCCs (Mercado et al., 2005; Uvarov et al., 2007), although its functional significance is unknown.

\section{C. elegans KCC-2 exhibits transport properties characteristic} of vertebrate KCCs

To test whether C. elegans KCC-2 is a potassium chloride cotransporter as suggested by phylogeny, we measured the functional properties of KCC-2. A previous functional characterization of $C$. elegans KCC-1 in HEK-293 cells resulted in a modest twofold increase in transport activity that was lower than, but consistent with, that observed with vertebrate KCCs (Holtzman et al., 1998). The activity of potassium chloride cotransporters can also be assayed by expressing them in Xenopus oocytes, exposing the oocytes to a hypotonic solution to stimulate KCC activity and then testing whether high extracellular chloride levels cause uptake of the potassium tracer ${ }^{86} \mathrm{Rb}$ (Mount et al., 1999; Mercado et al., 2000, 2005; Song et al., 2002). We exposed oocytes injected with C. elegans $k c c-2$ cRNA, rat $K c c 2$ RNA, or water to hypotonic solution containing $79 \mathrm{~mm}$ chloride and measured transport activity. C. elegans KCC-2 expression resulted in a 13-fold increase in $\mathrm{Rb}$ influx compared with the water-injected control oocytes (Fig. 3A), whereas expression of rat KCC2 resulted in a 33-fold increase in $\mathrm{Rb}$ uptake compared with the water-injected control (Fig. 3B). Our results demonstrate that both KCCs are active for uptake of a potassium tracer.

Isotonic conditions are not sufficient to activate vertebrate KCC1, KCC3, and KCC4 (Mount et al., 1999; Mercado et al., 2000), and $C$. elegans KCC-2 did not exhibit significant transport activity when exposed to isotonic solution (Fig. 3A). Oocytes injected with rat KCC2 exhibited a 7.2-fold increase in Rb uptake compared with water-injected oocytes in isotonic solution, and hypotonic solution caused a further 3.4-fold increase in Rb uptake (Fig. 3B). C. elegans KCC-2 likely does not exhibit transport activity in isotonic solution because it lacks the specific C-terminal domain that mediates constitutive transport by vertebrate KCC2 (Mercado et al., 2006).

KCCs require chloride in the extracellular solution to drive $\mathrm{Rb}$ influx (Payne, 1997; Mount et al., 1999; Mercado et al., 2000; 

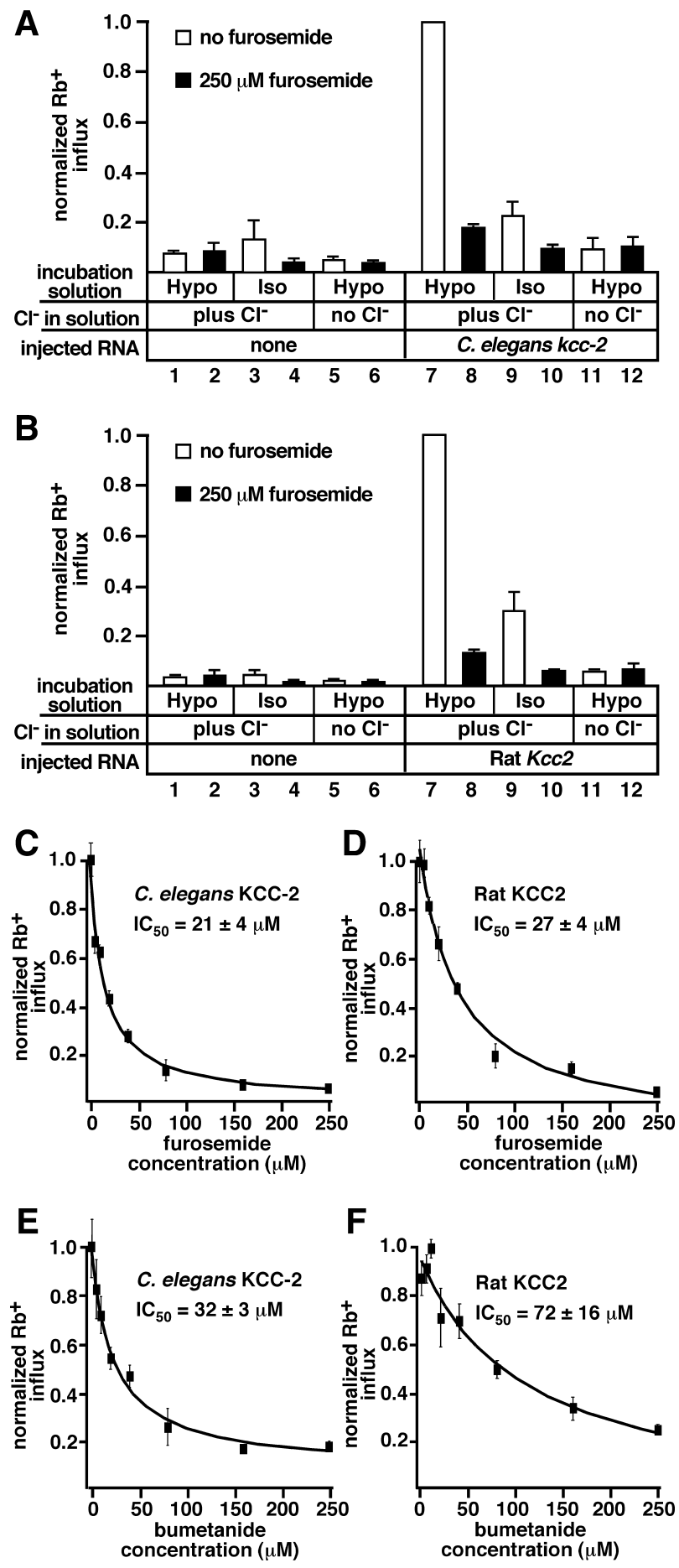

Figure 3. C. elegans KCC-2 exhibits transport properties similar to those of vertebrate potassium chloride cotransporters. $\boldsymbol{A}, \boldsymbol{B}$, Xenopus oocytes expressing $C$. elegans $k c c-2$, rat $K c c 2$, or injected with water (none) were tested for uptake of the potassium tracer ${ }^{86} \mathrm{Rb}$ after exposure

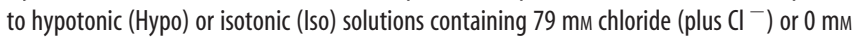
chloride $\left(\mathrm{no} \mathrm{Cl}^{-}\right)$. Measurements in the presence of $250 \mu \mathrm{m}$ furosemide were used to detect the background from furosemide-insensitive transporters endogenous to Xenopus oocytes. $\boldsymbol{A}, \boldsymbol{C}$. elegans KCC-2 transport activity was stimulated by hypotonic solution (compare 1 with 7). Isotonic conditions did not stimulate activity (compare 3 with 9). The loop diuretic furosemide inhibited KCC-2 activity (compare 7 with 8). KCC -2 did not uptake ${ }^{86} \mathrm{Rb}$ in the absence of extracellular chloride (compare 7 with 11). Bars $1-6$ and $8-12$ are significantly different from $7 ; p<0.01$. Bars $8-12$ are not significantly different from the respective water-injected controls (bars $2-6$ ). C. elegans $\mathrm{KCC}-2$ expression resulted in the influx of $2.90 \pm 0.51 \mathrm{nmol} \mathrm{Rb} / \mathrm{h}$ per oocyte. Statistical analysis of non-normalized values resulted in the same significance results. $\boldsymbol{B}$, Rat KCC2 transport activity was stimulated by hypotonic solution (compare 1 with 7), inhibited
Song et al., 2002). In the absence of chloride, oocytes expressing C. elegans KCC-2 (Fig. 3A) or rat KCC2 (Fig. 3B) did not exhibit significant ${ }^{86} \mathrm{Rb}$ uptake compared with the water-injected controls, indicating this potassium tracer is cotransported with chloride.

The loop diuretics furosemide and bumetanide inhibit KCC activity (Payne, 1997; Mercado et al., 2000; Song et al., 2002). Application of $250 \mu \mathrm{M}$ furosemide significantly inhibited both $C$. elegans KCC-2 and rat KCC2 (Fig. $3 A, B$ ). We performed doseresponse experiments to determine the $C$. elegans KCC-2 and rat KCC2 $\mathrm{IC}_{50}$ values for furosemide and bumetanide. The C. elegans KCC-2 IC $_{50}$ for furosemide was $21 \pm 4 \mu \mathrm{M}$ (Fig. 3C), not significantly different from the rat KCC2 $\mathrm{IC}_{50}$ for furosemide $(27 \pm 4$ $\mu \mathrm{M})$ (Fig. 3D). C. elegans KCC-2 and rat KCC2 $\mathrm{IC}_{50}$ values for bumetanide were $32 \pm 3$ and $72 \pm 16 \mu \mathrm{M}$, respectively (Fig. $3 E$, $F$ ), and both were an order of magnitude higher than those reported for vertebrate NKCCs (Plata et al., 2002).

\section{C. elegans $k c c-2$ is widely expressed in muscles and neurons}

To examine the $k c c-2$ expression pattern, we developed multiple $k c c-2$ promoter::gfp::kcc-2 $3^{\prime} \mathrm{UTR}$ reporter transgenes, which contained the $5^{\prime}$ promoter and $3^{\prime}$ untranslated region sequences for the three different $k c c-2$ isoforms driving expression of the GFP (supplemental Fig. 5A, available at www.jneurosci.org as supplemental material). We found that the $k c c-2 b$ promoter drove expression of $g f p$ in head, tail, and ventral cord motor neurons as well as body wall muscles, egg-laying muscles, uv1 uterus-associated endocrine cells, the distal tip cell, and the intestine (Fig. $4 A-C$ ). Using the $k c c-2 c$ promoter::gfp::kcc-2 3' UTR reporter transgene, we observed $\mathrm{KCC}-2$ reporter expression in the HSN neuron and utse uterine seam cell as well as many neurons in the head and tail (Fig. $4 D-F$ ). The $k c c-2 a$ promoter drove expression of $g f p$ in a few head and tail neurons (data not shown). A summary of the $k c c-2$ expression pattern can be found in supplemental Figure $5 B$ (available at www.jneurosci.org as supplemental material). Our analysis shows that C. elegans KCC-2 is predominantly expressed in neurons and muscles, consistent with the possibility that it functions to support inhibition of these cells.

\section{KCC-2 supports inhibitory neurotransmission through} ligand-gated chloride channels

We hypothesized that C. elegans KCC-2, like mammalian KCC2, may establish chloride gradients necessary for the inhibitory effects of neurotransmitter-gated chloride channels. We tested this by treating C. elegans with agonists for such channels and assaying for behavioral effects.

The C. elegans $\mathrm{GABA}_{\mathrm{A}}$ receptor UNC-49 is a GABA-gated chloride channel found on body-wall muscles that allows GABAergic motor neurons to relax the muscles on one side of the

by the loop diuretic furosemide (compare 7 with 8 ), and required extracellular chloride (compare 7 with 11). Rat KCC2 exhibits significant transport under isotonic conditions (compare 3 with $9 ; p<0.05$ ), although greater transport activity was observed in hypotonic solution (compare 7 with 9). Bars $1-6$ and $8-12$ are significantly different from 7; $p<0.01$. Expression of rat KCC2 resulted in the influx of $6.96 \pm 0.69 \mathrm{nmol} \mathrm{Rb} / \mathrm{h}$ per 00 cyte. In $\boldsymbol{A}$ and $\boldsymbol{B}$, values were normalized to uptake by the transporters under hypotonic conditions plus chloride; error bars show SE. $\boldsymbol{C}-\boldsymbol{F}$, Oocytes expressing C. elegans KCC-2 $(\boldsymbol{C}, \boldsymbol{E})$ or rat KCC2 $(\boldsymbol{D}, \boldsymbol{F})$ were exposed to increasing concentrations of furosemide $(\boldsymbol{C}, \boldsymbol{D})$ or bumetanide $(\boldsymbol{E}, \boldsymbol{F})$, from 0 to $250 \mu \mathrm{m}$ in the ${ }^{86} \mathrm{Rb}$ flux solution. The $\mathrm{IC}_{50}$ value is an average from three experiments, $\pm \mathrm{SE} ; \boldsymbol{C}-\boldsymbol{F}$ show representative experiments. Values were normalized to maximum ${ }^{86} \mathrm{Rb}$ influx in each experiment; error bars indicate SE. 
body at a time, enabling coordinated body bends (Bamber et al., 1999). Treating wild-type worms with the $\mathrm{GABA}_{\mathrm{A}}$ agonist muscimol inhibits contraction of all muscle cells simultaneously, so that animals respond to prodding with an uncoordinated contraction/relaxation response known as the "rubber-band" phenotype (de la Cruz et al., 2003). We found that $u n c-49$ and $k c c-2$ mutants failed to show the rubber-band phenotype when prodded after muscimol treatment (Fig. 5A). This suggests that KCC-2, which is expressed in body-wall muscles (Fig. 4B), may establish the chloride gradient required for the inhibitory action of UNC-49 in these cells.

We found two more examples of inhibitory signaling that depend on KCC-2. While analyzing the effect of muscimol on locomotion, we noticed that muscimol dramatically inhibited egg laying, suggesting a novel role for GABA in the regulation of egg-laying behavior. We quantitated this by counting the eggs laid by animals on plates lacking or containing $0.5 \mathrm{~mm}$ muscimol. In contrast to the $99 \%$ inhibition of egg laying seen in the wild type, muscimol caused only a $9 \%$ reduction in egg laying in the $u n c-49$ mutant and only a $14 \%$ reduction in the $k c c-2$ mutant (Fig. $5 B)$. Thus, inhibition of egg laying by $\mathrm{GABA}_{\mathrm{A}}$ receptor signaling is dependent on KCC-2.

Finally, we found that inhibition of locomotion by the serotonin-gated chloride channel MOD-1 also depends on KCC-2.

Serotonin treatment paralyzes wild-type animals, whereas mod-1 mutants are defective for this response (Ranganathan et al., 2000). We found that $k c c-2$ mutants are partially defective for this response to serotonin (supplemental Fig. 6, available at www. jneurosci.org as supplemental material). The partial nature of the defect suggests that KCC-2 may act with other chloride transporters to establish the chloride gradient required for MOD-1 function, although the other C. elegans KCCs do not appear to act redundantly with KCC-2 to support MOD-1 function (supplemental Fig. 7, available at www.jneurosci.org as supplemental material) (data not shown). Alternatively, in the absence of KCC-2, activation of MOD-1 may no longer have a hyperpolarizing effect, but shunting of membrane conductance may still occur to cause partial inhibition.

Chloride gradients appear to be altered in the $k c c-2$ mutant Mammalian cells that do not express KCC2 can have reversed chloride gradients, such that opening chloride channels causes depolarizing rather than hyperpolarizing responses (Rivera et al., 1999; Hübner et al., 2001). For example, in immature neurons, before the onset of KCC2 expression, NKCC1 drives chloride influx, causing intracellular chloride levels to remain high. As a result, activation of neurotransmitter-gated chloride channels causes chloride efflux, resulting in a depolarizing response (Fig. $5 C$ ). In mature neurons, high levels of KCC2 are present, and the potassium gradient is used to drive efflux of potassium and chlo- $k c c-2 b$ isoform promoter::gfp
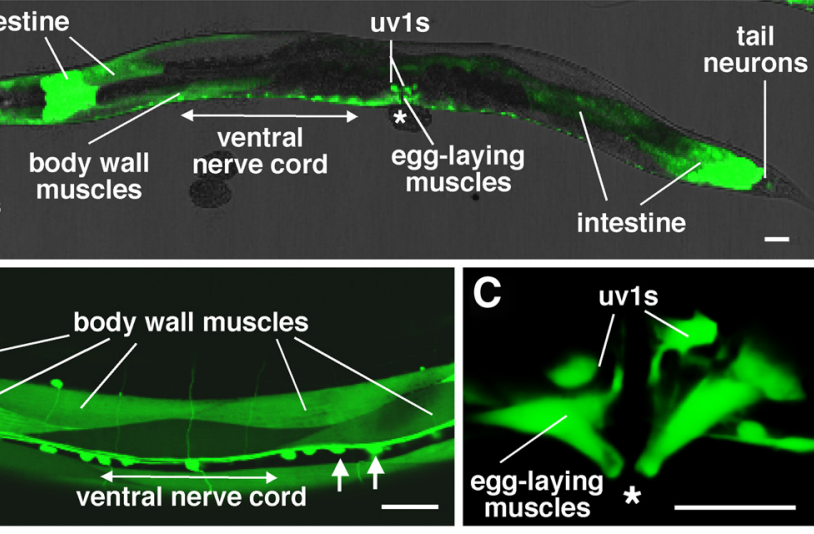

$k c c-2 c$ isoform promoter::gfp
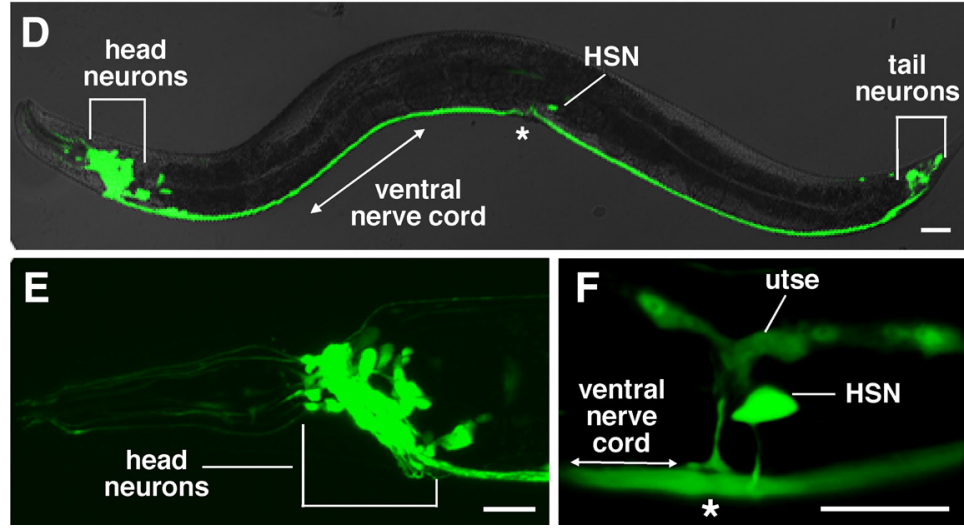

Figure 4. C. elegans KCC-2 is predominantly expressed in neurons and muscles. $\boldsymbol{A}-\boldsymbol{C}$, GFP fluorescence in animals carrying a kcc-2b promoter::gfp::kcc-23' UTR reporter transgene. This reporter transgene was expressed in many cells, including body-wall scles and ventral cord motor neurons (arrows) (B), as well as egg-laying muscles and uv1 cells (C). $\boldsymbol{D}-\boldsymbol{F}$, GFP fluorescence in animals carrying a kcc-2c promoter::gfp::kcc-2 3' UTR reporter transgene. This reporter transgene was expressed in head and tail neurons $(\boldsymbol{E})$ as well as the HSN neurons and utse $(\boldsymbol{F})$. Scale bar, $20 \mu \mathrm{m}$. Asterisks indicate the position of the vulva.

ride. This lowers intracellular chloride concentration such that activation of neurotransmitter-gated chloride channels causes neuronal inhibition (Fig. 5D).

In an analogous finding, we observed that activation of the UNC-49 GABA receptor has an excitatory effect on body-wall muscles of $k c c-2$ mutants rather than the inhibitory effect it has in the wild type. Simultaneous contraction of all body-wall muscles shortens the body of C. elegans, whereas relaxation of these muscles lengthens the body (Liewald et al., 2008). We placed animals on plates either lacking or containing the $\mathrm{GABA}_{\mathrm{A}}$ agonist muscimol and measured body length to determine whether UNC-49 activation caused muscle contraction or relaxation (Fig. 5E,F). In the wild type, exposure to muscimol resulted in a significant increase in body length, from $926.5 \pm 4.5 \mu \mathrm{m}$ on the control plates to $1000.8 \pm 5.3 \mu \mathrm{m}$ on the muscimol plates (Fig. $5 G$ ). In $k c c-2$ mutants, the effect of muscimol was reversed and a significant decrease in body length was observed, from $883.6 \pm 4.4 \mu \mathrm{m}$ on control plates to $820 \pm 6.6 \mu \mathrm{m}$ on muscimol plates (Fig. $5 G$ ). Exposure to muscimol did not affect the body length of the unc-49 mutant or the $k c c-2$; unc-49 double mutant (Fig. 5G). Thus, muscimol causes UNC-49-dependent relaxation of bodywall muscles in the wild type and UNC-49-dependent contraction of body-wall muscles in the $k c c-2$ mutant.

Because UNC-49 is expressed in the body-wall muscles but not the neurons that innervate them (Bamber et al., 1999), muscimol likely acts directly on the body-wall muscles. To test this, 

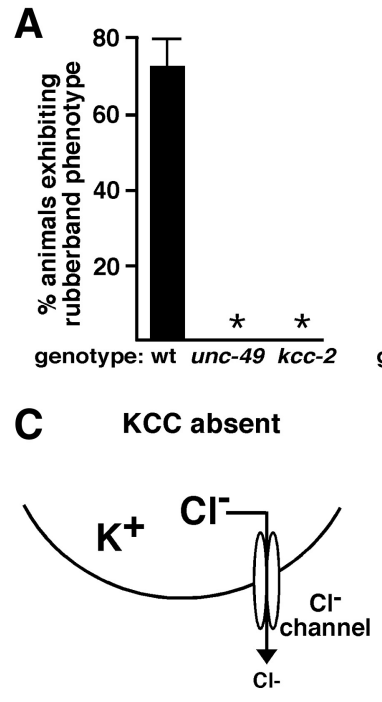

depolarization
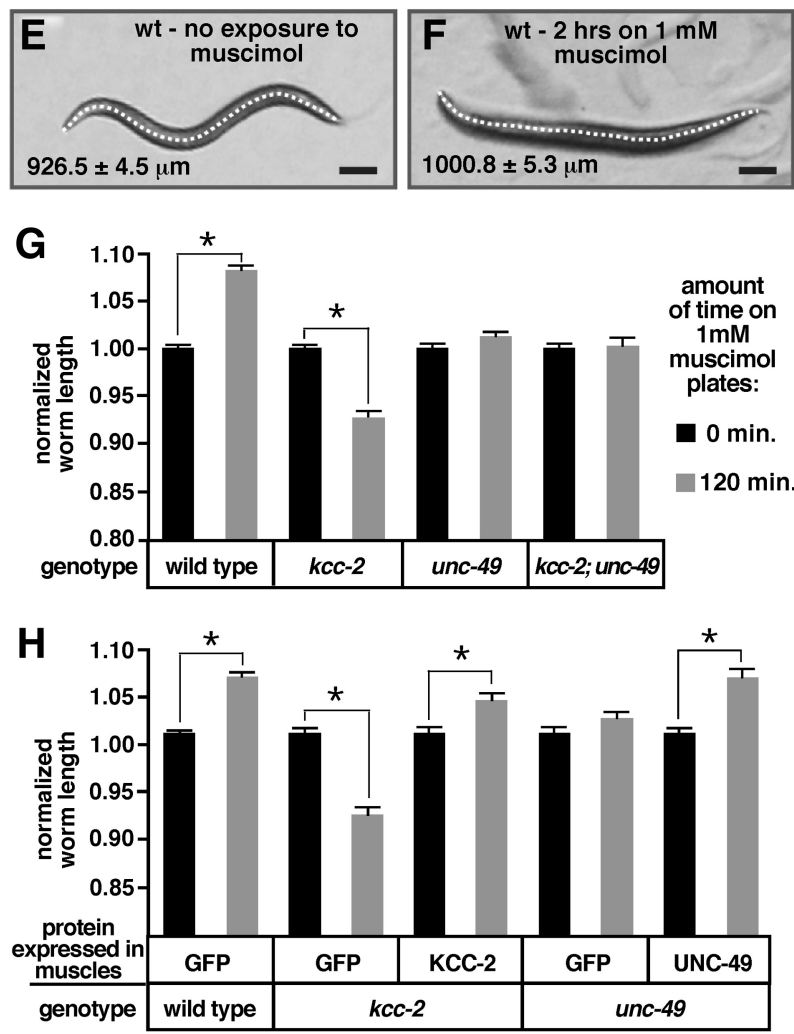

amount of time on $1 \mathrm{mM}$ muscimol plates:
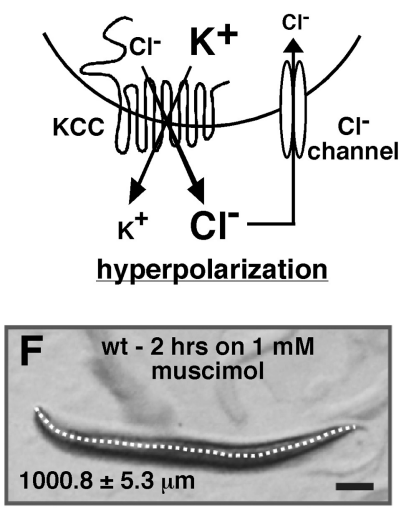

hyperpolarization

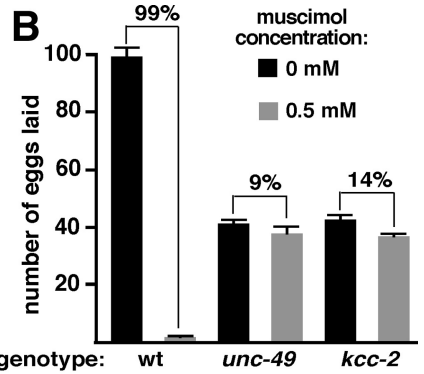

D KCC present

.
adult stage (Adler et al., 2006; Ding et al., 2007). Twelve hours after the late L4 stage, serotonin is released from the functionally mature HSNs as the first eggs are laid (Jose et al., 2007). To determine whether $k c c-2$ expression levels change in the HSNs during development, we used the $k c c-2 c$ promoter:: $g f p:: k c c-23^{\prime}$ UTR reporter, which is the only $k c c-2$ isoform-specific reporter transgene expressed in the HSNs, and analyzed GFP fluorescence levels at the L3, L4, and adult stages. Twenty-nine of $30 \mathrm{~L} 3$ animals did not exhibit detectable GFP fluorescence in the HSN neurons, whereas all L4 animals showed low but detectable levels of GFP expression in the HSNs. An additional 5.9-fold increase in GFP fluorescence intensity in the HSN was observed in adults compared with the L4 animals (Fig. 6A-D). Although our GFP reporter does not measure endogenous $k c c-2$ mRNA levels, our analysis indicates that $k c c-2$ is not expressed in the HSN before synapse formation, weakly expressed in the HSNs during initial synapse formation, further upregulated during the final remodeling of the HSN synapses, and strongly expressed in the functionally mature neuron. This is analogous to the increase in expression of $K c c 2$ observed during mammalian synaptogenesis.

\footnotetext{
$\leftarrow$

$\mathrm{KCCS}$ use the potassium $\left(\mathrm{K}^{+}\right)$concentration gradient to drive electroneutral extrusion of $\mathrm{K}^{+}$ and $\mathrm{Cl}^{-}$to establish $\mathrm{Cl}^{-}$concentration gradient, with higher extracellular $\mathrm{Cl}^{-}$. Activation of $\mathrm{a} \mathrm{Cl}^{-}$channel allows $\mathrm{Cl}^{-}$to reenter the neuron, resulting in neural inhibition. $\boldsymbol{E}, \boldsymbol{F}$, Representative wild-type animals in the absence $(\boldsymbol{E})$ and presence $(\boldsymbol{F})$ of muscimol. A line was drawn down the center of each animal to measure body length. Average body length for each condition is indicated. Scale bars, $100 \mu \mathrm{m}$. G, Exposure to muscimol caused a significant increase in body length of wild-type animals, a decrease in the length of the $k c c-2$ mutant, and no change in the unc- 49 single mutant or the $k c c-2$; unc- 49 double mutant. $\boldsymbol{H}$, After reexpression of $k c c-2$ in the body-wall muscles of the $k c c-2$ mutant or reexpression of unc- 49 in the body-wall muscles of the unc- 49 mutant, muscimol caused a significant increase in body length, as observed in wild-type animals. $\boldsymbol{G}, \boldsymbol{H}$, For each genotype, measurements were normalized to body length in the absence of muscimol. Error bars show SE, and asterisks indicate a significant difference $(p<0.0001)$.
}

Figure 5. KCC-2 is required for the inhibitory effects of ligand-gated chloride channels. $\boldsymbol{A}$, Exposure to $1 \mathrm{~mm}$ muscimol induced a rubber-band phenotype (quick contraction and relaxkcc-2 mutants $n>110$ for each asterisks indicate values significantly different from the wild type $(p<0.05)$. $\boldsymbol{B}$, The average number of eggs laid by five animals for $2 \mathrm{~h}$ on plates in the presence or absence of $0.5 \mathrm{~mm}$ muscimol. Animals with mutations in unc- 49 and $k c c-2$ were resistant to the inhibitory effects of muscimol on egg-laying behavior. Brackets are labeled with the percentage inhibition of egg laying induced by muscimol. The unc- 49 and $k c c-2$ mutants retained significantly fewer eggs in utero compared with the wild type as a result of a decrease in egg production and thus laid fewer eggs on the non-muscimol plates. For each genotype and condition, $n=30$ animals, and error bars show SE. C, In the absence of $\mathrm{KCC}$ activity, intracellular $\mathrm{Cl}^{-}$levels remain high, and stimulation of a $\mathrm{Cl}^{-}$channel causes $\mathrm{Cl}^{-}$to move out of the neuron, leading to depolarization. $\boldsymbol{D}$, mine whether this could rescue the muscimol response defects. Exposure to muscimol caused a significant decrease in body length in the $k c c-2$ mutant, but, after reexpression of KCC-2 in
the body-wall muscles, muscimol exposure caused a significant increase in body length, similar to that seen in the wild type (Fig. $5 H)$. In unc-49 mutants, muscimol exposure did not cause a UNC-49 in the body-wall muscles, muscimol caused a significant increase in body length (Fig. $5 H$ ). These results indicate that sufficient to mediate the inhibitory effects of muscimol on locomotion.

\section{$k c c-2$ expression is transcriptionally upregulated during HSN development}

In mammals, Kcc2 expression is transcriptionally upregulated in
the final stages of neuronal development (Rivera et al., 1999), and we found that $C$. elegans $k c c-2$ is upregulated during HSN develvelopment, whereas many other neurons in C. elegans are already functional and express $k c c-2$ in the first larval stage (L1). In $C$. elegans, the HSN neuron extends its axon during the L2 and L3 larval stages, and the synapses, which begin to form during the L4

we transgenically expressed KCC-2 and UNC-49 in the body-wall

\section{.}



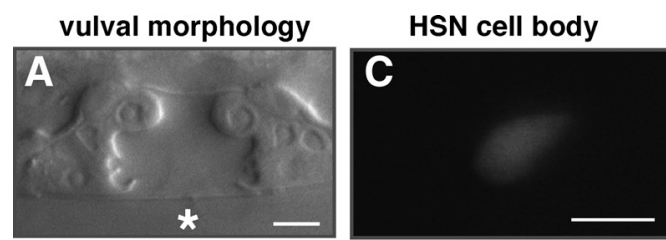

L4
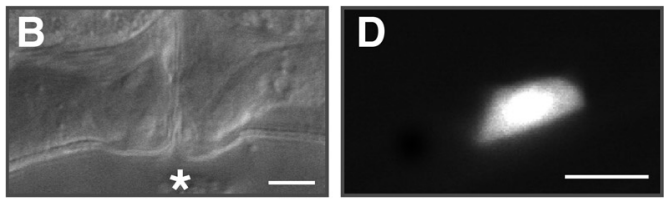

adult

E

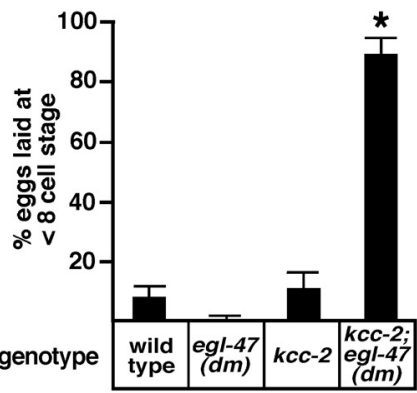

$\mathbf{F}$

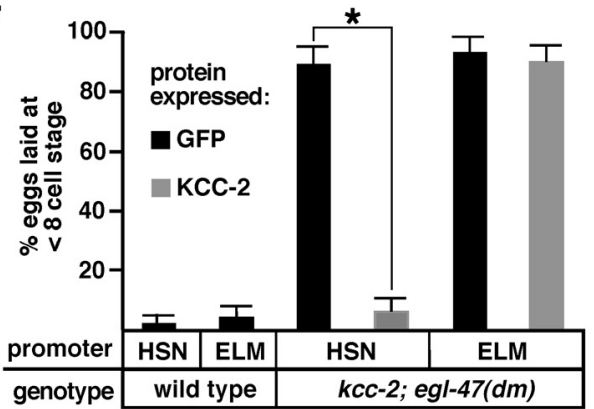

G

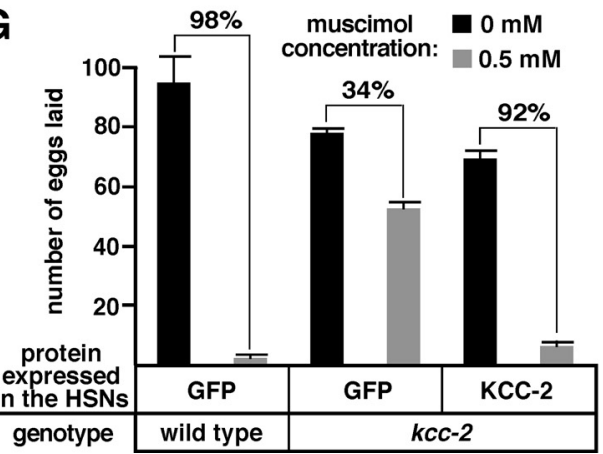

Figure 6. $k c c-2$ expression in the HSN neurons is developmentally upregulated and causes a shift in HSN activity. $A, B$, Bright-field images show $L 4$ and adult vulval morphology of wild-type animals, which was used to precisely determine developmental age. Asterisks indicate the position of the center of the vulva. $C, D, G F P$ expressed by the $k c c-2$ c promoter in HSN cell bodies from the same animals shown in $\boldsymbol{A}$ and $\boldsymbol{B}$, respectively. Scale bars, $5 \mu \mathrm{m}$. $\boldsymbol{E}$, The $k c c-2$; egl$47(\mathrm{dm})$ double mutant laid many early-stage eggs that had not developed beyond the eightcell stage, whereas such hyperactive egg laying was not seen in the $k c c-2$ or egl-47(dm) single mutants. $F$, Reexpression of $k c c-2$ in the HSN neurons, but not the ELMs of the $k c c-2 ;$ egl- $47(\mathrm{dm})$ mutant, rescued the synthetic hyperactive egg-laying behavior. For $\boldsymbol{E}$ and $\boldsymbol{F}, n \geq 100$ eggs per genotype, error bars show the $95 \%$ confidence interval, and asterisks indicate values significantly different from the respective controls $(p<0.05)$. $\mathbf{G}$, Reexpression of $k c c-2$ in the HSN neurons of the $k c c-2$ mutant restored the inhibition of muscimol on egg-laying behavior. Brackets are labeled with the percentage inhibition of egg laying induced by muscimol. For each genotype, $n=50$ animals (10 animals from five independent transgenic lines); error bars show SE.
Loss of $k c c-2$ reverses the effects of $e g l-47(\mathrm{dm})$ on HSN motor neuron output

The upregulation of C. elegans $k c c-2$ during HSN development may reduce intracellular chloride to shift the effects of neurotransmitter signaling onto the HSN from excitatory to inhibitory, analogous to the shift caused by upregulation of KCC2 in developing mammalian neurons. Because egg laying is the behavioral output of the HSN motor neurons, we used this behavior to measure HSN excitation versus inhibition by the HSN-expressed putative receptor EGL-47. The previously described early-stage egg assay was used to measure egg laying rates. In this assay, the egl-47 (dm) mutant and the $k c c-2$ single mutant, like the wild type, laid very few early-stage eggs (Fig. $6 E$ ). Surprisingly, the $k c c-2$; egl-47 (dm) double mutant showed strong hyperactive egg laying, because it laid $89 \%$ early-stage eggs (Fig. 6E). Thus, egl-47( $\mathrm{dm}$ ) inhibits egg laying in the wild-type background but dramatically stimulates egg laying in the $k c c-2$ mutant background.

Because egl-47( dm $)$ has its effect on egg laying by acting in the HSNs (Moresco and Koelle, 2004), we determined whether $k c c-2$ also functions in the HSNs to reverse the effect of egl-47 $(\mathrm{dm})$ on egg laying. We used cell-specific promoters to express $k c c-2$ in the HSNs or ELMs of the $k c c-2 ;$ egl-47 $(\mathrm{dm})$ mutant. Expression of $k c c-2$ in the HSNs, but not the ELMs, of the $k c c-2$; egl$47(\mathrm{dm})$ double mutant significantly reduced the number of early-stage eggs laid from 89 to $6 \%$ (Fig. $6 \mathrm{~F}$ ). This suggests that KCC-2 activity in the HSNs is sufficient to reverse the effect of egl-47(dm).

We also determined whether KCC-2 function in the HSNs mediates the inhibition of egg laying by the $\mathrm{GABA}_{\mathrm{A}}$ receptor agonist muscimol. Whereas muscimol caused a $98 \%$ inhibition of egg laying in the wild type, only a $34 \%$ reduction in egg laying was observed in the $k c c-2$ mutant (Fig. $6 G$ ). Expression of $k c c-2$ in the HSNs of the $k c c-2$ mutant restored the inhibition of egg laying to 92\% (Fig. 6G). Our results suggest that KCC-2 activity in the HSNs is sufficient to permit inhibition of egg laying by $\mathrm{GABA}_{\mathrm{A}}$ receptors, although there is currently no evidence that $u n c-49$ is expressed in the HSNs (Bamber et al., 1999) (data not shown).

\section{$k c c-2$ mutants exhibit a decrease in the synaptic vesicle population within $\mathrm{HSN}$ presynaptic termini}

Why might $k c c-2$ mutants show wild-type egg laying (Fig. $6 E$ ), despite being defective for the inhibitory effects of EGL-47 (Fig. $6 E$ ), GABA (Fig. 5B), and possibly other neurotransmitters on this behavior? We hypothesized that the HSN synapses of $k c c-2$ mutants might undergo changes to decrease their ability to release neurotransmitters that trigger egg laying, thus compensating for a lack of inhibition. The HSN process forms two to four presynaptic varicosities that contact the egg-laying muscles within $10 \mu \mathrm{m}$ of the vulval slit (Fig. 7A) (Tanis et al., 2008). To visualize these synapses, we used an HSN-specific promoter to transgenically coexpress the red fluorescent protein DsRed2 and GFP-tagged synaptobrevin (SNB-1::GFP). DsRed2 fills out the HSNs and was used to study overall neuron morphology as well as the structure and volume of the HSN presynaptic varicosities. SNB-1::GFP labels synaptic vesicles at presynaptic termini (Nonet, 1999) and was used to measure the size of the synaptic vesicle population within the HSN presynaptic varicosities. SNB-1::GFP labeling within the HSN presynaptic varicosities changes in mutants that exhibit abnormal HSN synapse development and correlates with changes in synaptic vesicles and active zones as determined by serial-section electron microscopy (Shen et al., 2004). The chromosomally integrated transgene expressing DsRed 2 and SNB-1::GFP in the HSN was crossed into wild-type 

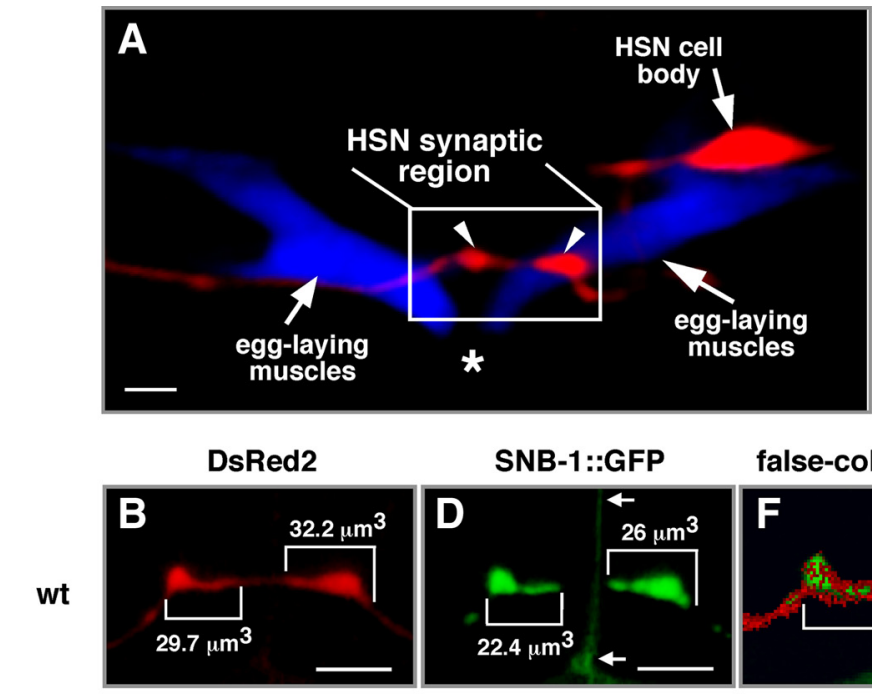

false-colored merge
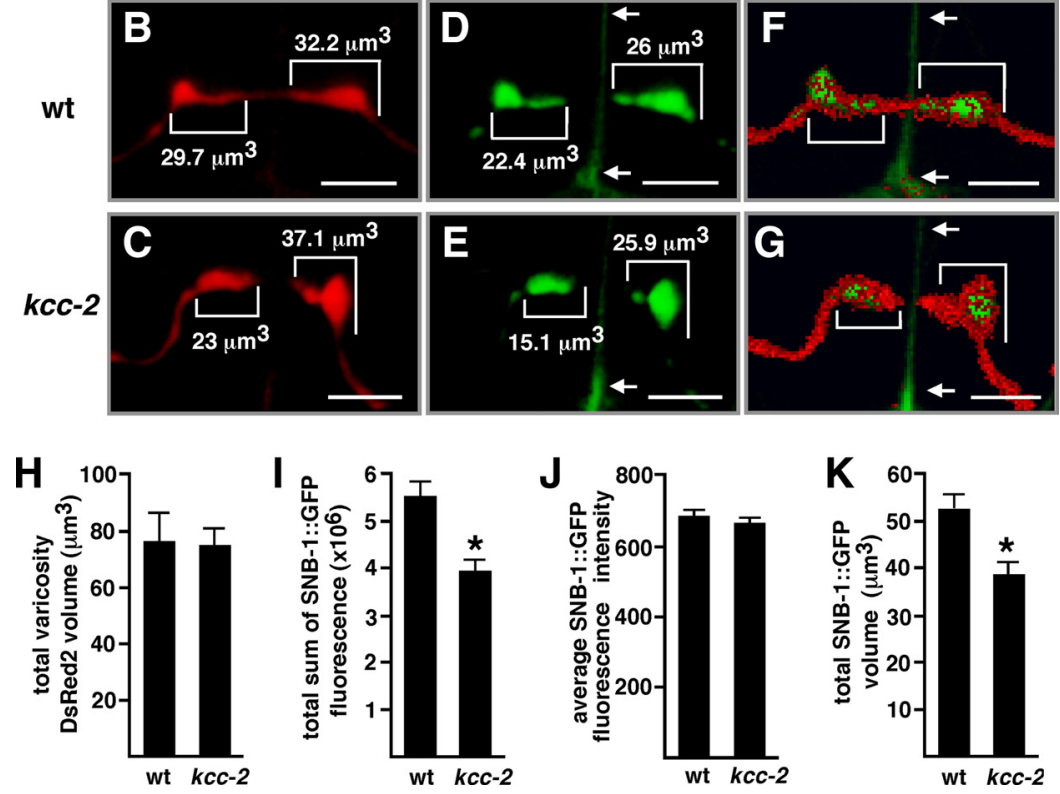

Figure 7. $k c c-2$ mutants exhibit a decrease in the size of the synaptic vesicle population within HSN presynaptic termini. $\boldsymbol{A}$, Confocal image of the HSNL neuron expressing the red fluorescent protein DsRed2 and the egg-laying muscles expressing the cyan fluorescent protein CFP. The HSN process forms presynaptic varicosities (arrowheads) that contact the egg-laying muscles near the vulva. Scale bar, $5 \mu \mathrm{m}$. Asterisk indicates the position of vulva. $\boldsymbol{B}, \boldsymbol{C}$, Expression of DsRed2 in wild-type (wt) (B) and kcc-2 mutant (C) HSNs. D, $\boldsymbol{E}$, Expression of SNB-1::GFP in wild-type (D) and kcc-2 mutant (E) HSNs. In $\boldsymbol{B}$ and $\boldsymbol{C}$, numbers indicate the DsRed2 volume for each varicosity, and, in $\boldsymbol{D}$ and $\boldsymbol{E}$, numbers indicate the volume of the SNB-1::GFP-labeled structure within each varicosity. Images in $\boldsymbol{B}$ - $\boldsymbol{E}$ are two-dimensional representations of three-dimensional images in which each two-dimensional pixel is shown at the brightest individual voxel intensity from the stack of three-dimensional voxels that it represents. $F, G$, Merged images in which the SNB-1::GFP is represented as in $\boldsymbol{D}$ and $\boldsymbol{E}$, but DsRed2 pixels above background that do not overlap with the SNB-1::GFP are false colored red, partially obscuring the green label. These images emphasize that the perimeters of the synaptic varicosities are labeled by DsRed2, and that the SNB-1::GFP-labeled structures are contained within the varicosities. The red-only perimeter is more prominent in kcc-2 than in the wild-type because of the decreased volume of SNB-1::GFP labeling in $k c c-2$. $\boldsymbol{B}-\mathbf{G}$, Brackets indicate synaptic varicosities, and arrows point to an artifact in the GFP channel caused by the vulval slit. Scale bars, $5 \mu \mathrm{m}$. $\boldsymbol{H}$, No significant difference in total DsRed2 varicosity volume was observed between wild-type and $k c c-2$ mutant animals $(p=0.89)$.II, A significant difference in the total sum of SNB-1::GFP fluorescence in the HSN synaptic region was observed between the wild-type and $k c c-2$ mutant animals $(p<0.005)$. $J$, The average SNB-1::GFP fluorescence intensity within the GFP-labeled structures was not significantly different between the wild-type and $k c c-2$ mutant animals ( $p=0.40)$, suggesting that the density of synaptic vesicles within these structures is unchanged. $\boldsymbol{K}$, The total volume of SNB-1::GFP-labeled structures in the HSN synaptic region was significantly less in the $k c c-2$ mutant compared with the wild type $(p<0.005)$, indicating that the reduction in total SNB-1::GFP fluorescence is likely attributable to a smaller population of synaptic vesicles inside the HSN varicosities of the $k c c-2$ mutant. For $\boldsymbol{H}$ - $\boldsymbol{K}$, error bars indicate $S E ; n=29$ varicosities from 11 animals for the wild type, and $n=36$ varicosities from 12 animals for the kcc-2 mutant.

and $k c c-2$ mutant backgrounds, and three-dimensional confocal images of the HSN synaptic region were acquired and quantitatively analyzed (Fig. $7 B-G$ ).

HSN process morphology was not altered in $k c c-2$ mutants (Fig. $7 H$ ) (data not shown). The total volume of the DsRed2filled varicosities, as well as total DsRed2 fluorescence within the varicosities, was not altered in the $k c c-2$ mutant compared with the wild-type (Fig. 7H) (data not shown).
Analysis of SNB-1::GFP indicated that there was a significant decrease in the size of the synaptic vesicle population within HSN presynaptic termini of $k c c-2 \mathrm{mu}$ tants. Each synaptic varicosity contained a GFP-labeled structure representing synaptic vesicle clusters at multiple active zones (Shen et al., 2004), and there was no difference in the number of these structures in the wild type $(2.64 \pm 0.24)$ compared with the $k c c-2$ mutant $(3 \pm 0.28)$. However, the total amount of SNB-1::GFP fluorescence in the synaptic region of the $k c c-2$ mutant was $28.6 \%$ less than in the wild type (Fig. 7I). This significant difference in total SNB-1::GFP fluorescence was not attributable to a difference in average GFP intensity within the fluorescent structures comparing the wild-type and $k c c-2 \mathrm{mu}$ tant animals (Fig. $7 J$ ). Instead, the average total SNB-1::GFP volume in the $k c c-2$ mutant was $38.5 \pm 2.8 \mu \mathrm{m}^{3}$, significantly less than the $52.1 \pm 3.2 \mu \mathrm{m}^{3}$ average observed in the wild type (Fig. $7 K$ ). This decrease in the SNB-1::GFP volume in the $k c c-2$ mutant results in a greater fringe around the perimeter of the synaptic varicosities that is labeled only by DsRed2 (Fig. 7F, G). An analysis of GFP::SYD-2 to visualize active zones in the HSNs of wild-type and $k c c-2$ mutants showed no detectable difference in this active zone marker (data not shown). These results suggest that the $k c c-2$ HSN presynaptic terminus contains fewer synaptic vesicles, whereas overall presynaptic varicosity morphology and the formation of active zones remained unchanged.

\section{Discussion}

\section{KCC-2 is required for}

\section{inhibitory neurotransmission}

In this work, we show that $C$. elegans KCC-2 is required for the inhibitory effects of the $\mathrm{GABA}_{\mathrm{A}}$ receptor UNC-49, the serotonin-gated chloride channel MOD-1, and the transmembrane protein EGL-47. C. elegans KCC-2 exhibits potassium chloride cotransport activity in a heterologous system and has a physiological function analogous to that of mammalian KCC2. Both transporters appear to regulate intracellular chloride levels so that the opening of chloride channels leads to neuronal inhibition. We found that the effects of UNC-49 and EGL-47 become excitatory rather than inhibitory in the $k c c-2$ mutant background. This suggests that the cells in the $k c c-2$ mutant have a mechanism to drive chloride influx but not efflux, resulting in reversed chloride gradients.

The power of the egl-47( $\mathrm{dm})$ suppressor screen provides us with the opportunity to identify additional proteins that regulate chloride gradients and inhibitory neurotransmission in vivo. In- 
deed, just 7 of 28 mutations isolated in this screen are alleles of $k c c-2$, and the remaining mutations identify additional genes that may also be important for neural inhibition.

\section{C. elegans kcc-2 expression is} developmentally upregulated in the HSN neurons to enable inhibition of HSN activity

In the immature brain, the excitatory action of GABA regulates synapse formation and neuronal growth (Ben-Ari, 2002). The onset of KCC2 expression establishes a chloride gradient necessary for inhibitory signaling through chloride channels and causes a shift in GABA activity from excitatory to inhibitory (Rivera et al., 1999). In this work, we show that C. elegans $k c c-2$ expression in the HSNs commences in the L4 stage, the developmental stage during which HSN synapse formation initiates, and it is further upregulated by the time HSN synapse remodeling is complete in adult animals.

The genetic interaction between $k c c-2$ and $e g l-47(d m)$ demonstrates that the presence versus absence of KCC-2 causes a shift in HSN activity. Whereas egl$47(\mathrm{dm})$ inhibits adult HSN activity and thus egg laying in a wild-type background, it appears to induce HSN activity and stimulate hyperactive egg laying in the $k c c-2$ mutant background. Moresco and Koelle (2004) proposed that EGL-47 is an orphan G-proteincoupled receptor. However, this work shows that EGL-47 may function independently of the G-protein GOA-1 and that the effect of EGL-47 depends on the direction of the chloride gradient. Thus, we hypothesize that EGL-47(dm) may either be a constitutively active chloride channel or a transmembrane protein that activates chloride channels in the HSNs (Fig. 8). We note that EGL-47 is similar in sequence to insect gustatory receptors and to a novel light receptor, LITE-1, that allows C. elegans to respond to ultraviolet light (Edwards et al., 2008).

Our data suggest that upregulation of KCC expression to cause a shift from excitatory to inhibitory signaling through neurotransmitter-gated chloride channels is a general feature of neuron development. Upregulation of mammalian Kcc2 expression occurs over the several week period of synaptogenesis after birth (Ben-Ari, 2002), and we observed upregulation of C. elegans KCC-2 expression during HSN synaptogenesis. The correlation between the timing of KCC upregulation and this phase in development suggests that KCC activity may play an essential role in neural development and synapse formation. A clearer understanding of the purpose of KCC upregulation requires that it be studied in identified neurons that function in defined circuits that can be subjected to precise experimental manipulation. The C. elegans egg-laying circuit provides such a system.

\section{KCC-2 regulates synapse development to precisely balance} excitatory and inhibitory neurotransmission

A typical neural circuit with both excitatory and inhibitory synapses must undergo exquisitely coordinated development so that the mature circuit has the precise balance of excitatory and inhibitory signaling necessary to achieve the appropriate overall level of activity. Our results in the C. elegans egg-laying circuit suggest that an interplay between the development of inhibitory neurotransmitter signaling and the development of excitatory synapses is used to achieve this necessary balance.

KCC-2 upregulation during HSN synapse development is correlated with the onset of several inhibitory inputs on the egglaying circuit. We found that inhibition of egg laying by the UNC-49 GABA ${ }_{\mathrm{A}}$ receptor and EGL-47 require KCC-2 expression. In $k c c-2$ mutant adults, the signals that normally inhibit egg laying may instead be excitatory as a result of reversed chloride gradients. Remarkably, in the absence of challenges to the egglaying system, we have not detected changes in the egg-laying behavior of $k c c-2$ mutants compared with wild-type animals. It appears that either the inhibitory signals that depend on $k c c-2$ have little significance for egg-laying behavior under standard laboratory conditions or else the egg-laying circuit has an extraordinary mechanism for compensating for the lack of inhibitory signaling to produce a normal level of behavioral output.

By quantitatively analyzing images of the HSN presynaptic termini, we have seen evidence for a mechanism that may compensate for the lack of inhibitory signaling. We saw a decrease in the size of the synaptic vesicle population within the HSN presynaptic termini of the $k c c-2$ mutant. This change is quite remarkable and specific, because we found that mutants for the $\mathrm{G} \alpha$ o gene goa-1, which have grossly hyperactive egg laying attributable to changes in HSN activity, did not exhibit detectable changes in SNB-1::GFP labeling of HSN presynaptic termini 
(Tanis et al., 2008). The reduction in the HSN presynaptic vesicle population seen in $k c c-2$ mutants likely reduces the capacity of the HSN to release serotonin to stimulate egg laying, thus compensating for the lack of inhibition through neurotransmittergated chloride channels. Our results suggest that there is plasticity in HSN synapse formation that allows the HSN neurons to develop synapses of the appropriate size so that inhibitory and excitatory signaling are properly balanced within the mature egglaying circuit.

Parallel work performed in mammals also suggests that proper levels of KCC expression are required to establish correct neuron morphology and synaptic connections (Chudotvorova et al., 2005; Akerman and Cline, 2006; Cancedda et al., 2007; Li et al., 2007; Reynolds et al., 2008). A decrease in dendritic arbor size was observed after premature expression of KCC2 by in utero electroporation in mouse brain cortical neurons, and this change in neuronal morphology was dependent on the ion transport activity of KCC-2 (Cancedda et al., 2007). Another recent study showed that dendritic spines from KCC2 knock-out mice exhibited elongated filopodia-like morphology with a reduced number of functional synapses and that the interaction of the C-terminal domain of KCC2 with the cytoskeleton-associate protein 4.1 regulates dendrite morphology (Li et al., 2007). Thus, KCC2 appears to affect the development and morphology of dendritic synapses in mammals.

Our studies in C. elegans did not detect any effects of $k c c-2$ mutations on the overall morphology of the HSN neurons, and studies in mammals have not yet examined the effects of KCC2 activity on the synaptic vesicle population in presynaptic termini. Thus, although there are overall parallels between the effects of KCC function on neural development in C. elegans and mammals, there may also be differences between the systems.

\section{Two forms of inhibitory neurotransmission are integrated by the HSN neurons to regulate egg-laying behavior}

Neurotransmitters can inhibit neural activity by either opening chloride channels or activating G-protein-coupled receptors that signal through $\mathrm{G} \alpha_{\mathrm{o}}$ to inhibit neurotransmitter release. Both forms of inhibitory neurotransmission function in the HSN neurons to regulate C. elegans egg laying, providing a model to investigate the relationship between these two modes of neural inhibition.

Egg-laying behavior is under complex regulation in response to environmental conditions and egg availability. C. elegans adults dramatically downregulate egg laying in the absence of food, apparently to prevent their eggs from being laid in unfavorable conditions (Dong et al., 2000). Egg laying occurs in short bursts separated by intervals of $\sim 20$ min during which further egg laying is inhibited, preventing the entire supply of eggs from being laid in one place (Waggoner et al., 1998). Finally, young adults initially inhibit egg-laying behavior until their uterus is first filled with eggs (Jose et al., 2007). These effects may all be mediated by neurotransmitters that signal onto the HSNs through chloride channels and/or G-protein-coupled receptors (Fig. 8).

Some of the specific neurotransmitters and their receptors have been identified. The VCs (ventral cord type C neurons) release acetylcholine that acts, in part, through the G-proteincoupled receptor GAR-2 on the HSNs to inhibit egg laying (Bany et al., 2003). Serotonin released by the HSNs signals back onto the HSNs through $\mathrm{G} \alpha_{\mathrm{o}}$-coupled autoreceptors, inhibiting HSN activity and possibly accounting for the 20 min intervals between bursts of egg laying (Shyn et al., 2003). The uv1 neuroendocrine cells release tyramine, which may act on a tyramine-gated chlo- ride channel on the HSNs (M. Alkema, personal communication) to inhibit egg-laying until the uterus fills with eggs (Jose et al., 2007). In this work, we show a novel role for GABA, which acts on the $\mathrm{GABA}_{\mathrm{A}}$ receptor UNC-49 to inhibit egg laying. Thus, multiple signals, each carrying different information relevant to setting the optimal egg-laying rate, converge on the HSNs. Therefore, the signals are integrated to set the level of HSN activity to control egg-laying behavior (Fig. 8).

\section{References}

Adler CE, Fetter RD, Bargmann CI (2006) UNC-6/Netrin induces neuronal asymmetry and defines the site of axon formation. Nat Neurosci 9:511-518.

Akerman CJ, Cline HT (2006) Depolarizing GABAergic conductances regulate the balance of excitation to inhibition in the developing retinotectal circuit in vivo. J Neurosci 26:5117-5130.

Bamber BA, Beg AA, Twyman RE, Jorgensen EM (1999) The Caenorhabditis elegans unc-49 locus encodes multiple subunits of a heteromultimeric GABA receptor. J Neurosci 19:5348-5359.

Bany IA, Dong MQ, Koelle MR (2003) Genetic and cellular basis for acetylcholine inhibition of Caenorhabditis elegans egg-laying behavior. J Neurosci 23:8060-8069.

Ben-Ari Y (2002) Excitatory actions of gaba during development: the nature of the nurture. Nat Rev Neurosci 3:728-739.

Brenner S (1974) The genetics of Caenorhabditis elegans. Genetics 77:71-94.

Cancedda L, Fiumelli H, Chen K, Poo MM (2007) Excitatory GABA action is essential for morphological maturation of cortical neurons in vivo. J Neurosci 27:5224-5235.

Caron L, Rousseau F, Gagnon E, Isenring P (2000) Cloning and functional characterization of a cation- $\mathrm{Cl}^{-}$cotransporter-interacting protein. J Biol Chem 275:32027-32036.

Chase DL, Koelle MR (2004) Genetic analysis of RGS protein function in Caenorhabditis elegans. Methods Enzymol 389:305-320.

Chudotvorova I, Ivanov A, Rama S, Hübner CA, Pellegrino C, Ben-Ari Y, Medina I (2005) Early expression of KCC2 in rat hippocampal cultures augments expression of functional GABA synapses. J Physiol 566:671-679.

Darman RB, Forbush B (2002) A regulatory locus of phosphorylation in the $\mathrm{N}$ terminus of the $\mathrm{Na}-\mathrm{K}-\mathrm{Cl}$ cotransporter, NKCC1. J Biol Chem 277:37542-37550.

de la Cruz IP, Levin JZ, Cummins C, Anderson P, Horvitz HR (2003) sup-9, sup-10, and unc-93 may encode components of a two-pore $\mathrm{K}^{+}$channel that coordinates muscle contraction in Caenorhabditis elegans. J Neurosci 23:9133-9145.

Ding M, Chao D, Wang G, Shen K (2007) Spatial regulation of an E3 ubiquitin ligase directs selective synapse elimination. Science 317:947-951.

Dong MQ, Chase D, Patikoglou GA, Koelle MR (2000) Multiple RGS proteins alter neural $\mathrm{G}$ protein signaling to allow C. elegans to rapidly change behavior when fed. Genes Dev 14:2003-2014.

Edwards SL, Charlie NK, Milfort MC, Brown BS, Gravlin CN, Knecht JE, Miller KG (2008) A novel molecular solution for ultraviolet light detection in Caenorhabditis elegans. PLoS Biol 6:e198.

Etchberger JF, Hobert O (2008) Vector-free DNA constructs improve transgene expression in C. elegans. Nat Methods 5:3.

Farrant M, Kaila K (2007) The cellular, molecular and ionic basis of GABA(A) receptor signalling. Prog Brain Res 160:59-87.

Gillen CM, Brill S, Payne JA, Forbush B $3^{\text {rd }}$ (1996) Molecular cloning and functional expression of the $\mathrm{K}-\mathrm{Cl}$ cotransporter from rabbit, rat, and human. A new member of the cation-chloride cotransporter family. J Biol Chem 271:16237-16244.

Holtzman EJ, Kumar S, Faaland CA, Warner F, Logue PJ, Erickson SJ, Ricken G, Waldman J, Kumar S, Dunham PB (1998) Cloning, characterization, and gene organization of $\mathrm{K}-\mathrm{Cl}$ cotransporter from pig and human kidney and C. elegans. Am J Physiol 275:F550-F564.

Huberfeld G, Wittner L, Clemenceau S, Baulac M, Kaila K, Miles R, Rivera C (2007) Perturbed chloride homeostasis and GABAergic signaling in human temporal lobe epilepsy. J Neurosci 27:9866-9873.

Hübner CA, Stein V, Hermans-Borgmeyer I, Meyer T, Ballanyi K, Jentsch TJ (2001) Disruption of KCC2 reveals an essential role of $\mathrm{K}-\mathrm{Cl}$ cotransport already in early synaptic inhibition. Neuron 30:515-524.

Jose AM, Bany IA, Chase DL, Koelle MR (2007) A specific subset of transient receptor potential vanilloid-type channel subunits in Caenorhabditis el- 
egans endocrine cells function as mixed heteromers to promote neurotransmitter release. Genetics 175:93-105.

Li H, Khirug S, Cai C, Ludwig A, Blaesse P, Kolikova J, Afzalov R, Coleman SK, Lauri S, Airaksinen MS, Keinänen K, Khiroug L, Saarma M, Kaila K, Rivera C (2007) KCC2 interacts with the dendritic cytoskeleton to promote spine development. Neuron 56:1019-1033.

Liewald JF, Brauner M, Stephens GJ, Bouhours M, Schultheis C, Zhen M, Gottschalk A (2008) Optogenetic analysis of synaptic function. Nat Methods 5:895-902.

Liu Z, Neff RA, Berg DK (2006) Sequential interplay of nicotinic and GABAergic signaling guides neuronal development. Science 314:1610-1613.

Mello CC, Kramer JM, Stinchcomb D, Ambros V (1991) Efficient gene transfer in C. elegans: extrachromosomal maintenance and integration of transforming sequences. EMBO J 10:3959-3970.

Mercado A, Song L, Vazquez N, Mount DB, Gamba G (2000) Functional comparison of the $\mathrm{K}^{+}-\mathrm{Cl}^{-}$cotransporters $\mathrm{KCCl}$ and $\mathrm{KCC}$. J Biol Chem 275:30326-30334.

Mercado A, Vázquez N, Song L, Cortés R, Enck AH, Welch R, Delpire E, Gamba G, Mount DB (2005) NH2-terminal heterogeneity in the KCC3 $\mathrm{K}^{+}-\mathrm{Cl}^{-}$cotransporter. Am J Physiol Renal Physiol 289:F1246-F1261.

Mercado A, Broumand V, Zandi-Nejad K, Enck AH, Mount DB (2006) A C-terminal domain in $\mathrm{KCC} 2$ confers constitutive $\mathrm{K}^{+}-\mathrm{Cl}^{-}$cotransport. J Biol Chem 281:1016-1026.

Mody I, De Koninck Y, Otis TS, Soltesz I (1994) Bridging the cleft at GABA synapses in the brain. Trends Neurosci 17:517-525.

Moresco JJ, Koelle MR (2004) Activation of EGL-47, a G $\alpha_{\mathrm{o}}$-coupled receptor, inhibits function of hermaphrodite-specific motor neurons to regulate Caenorhabditis elegans egg-laying behavior. J Neurosci 24:8522-8530.

Mount DB, Mercado A, Song L, Xu J, George AL Jr, Delpire E, Gamba G (1999) Cloning and characterization of KCC3 and KCC4, new members of the cation-chloride cotransporter gene family. J Biol Chem 274:16355-16362.

Nonet ML (1999) Visualization of synaptic specializations in live C. elegans with synaptic vesicle protein-GFP fusions. J Neurosci Methods 89:33-40.

Owens DF, Kriegstein AR (2002) Is there more to GABA than synaptic inhibition?. Nat Rev Neurosci 3:715-727.

Payne JA (1997) Functional characterization of the neuronal-specific K-Cl cotransporter: implications for $\left[\mathrm{K}^{+}\right]_{\mathrm{o}}$ regulation. Am J Physiol Cell Physiol 273:C1516-C1525.

Payne JA, Stevenson TJ, Donaldson LF (1996) Molecular characterization of a putative $\mathrm{K}-\mathrm{Cl}$ cotransporter in rat brain. A neuronal-specific isoform. J Biol Chem 271:16245-16252.

Plata C, Meade P, Vazquez N, Hebert SC, Gamba G (2002) Functional properties of the apical $\mathrm{Na}^{+}-\mathrm{K}^{+}-2 \mathrm{Cl}^{-}$cotransporter isoforms. J Biol Chem 277:11004-11012.
Ranganathan R, Cannon SC, Horvitz HR (2000) MOD-1 is a serotoningated chloride channel that modulates locomotory behaviour in C. elegans. Nature 408:470-475.

Reynolds A, Brustein E, Liao M, Mercado A, Babilonia E, Mount DB, Drapeau P (2008) Neurogenic role of the depolarizing chloride gradient revealed by global overexpression of KCC2 from the onset of development. J Neurosci 28:1588-1597.

Rivera C, Voipio J, Payne JA, Ruusuvuori E, Lahtinen H, Lamsa K, Pirvola U, Saarma M, Kaila K (1999) The $\mathrm{K}^{+} / \mathrm{Cl}^{-}$co-transporter KCC2 renders GABA hyperpolarizing during neuronal maturation. Nature 397:251-255.

Rivera C, Voipio J, Thomas-Crusells J, Li H, Emri Z, Sipilä S, Payne JA, Minichiello L, Saarma M, Kaila K (2004) Mechanism of activitydependent downregulation of the neuron-specific $\mathrm{K}-\mathrm{Cl}$ cotransporter KCC2. J Neurosci 24:4683-4691.

Shen K, Fetter RD, Bargmann CI (2004) Synaptic specificity is generated by the synaptic guidepost protein SYG-2 and its receptor, SYG-1. Cell 116:869-881.

Shyn SI, Kerr R, Schafer WR (2003) Serotonin and Go modulate functional states of neurons and muscles controlling C. elegans egg-laying behavior. Curr Biol 13:1910-1915.

Song L, Mercado A, Vázquez N, Xie Q, Desai R, George AL Jr, Gamba G, Mount DB (2002) Molecular, functional, and genomic characterization of human KCC2, the neuronal K-Cl cotransporter. Brain Res Mol Brain Res 103:91-105.

Tanis JE, Moresco JJ, Lindquist RA, Koelle MR (2008) Regulation of serotonin biosynthesis by the $\mathrm{G}$ proteins $\mathrm{G} \alpha_{\mathrm{o}}$ and $\mathrm{G} \alpha_{\mathrm{q}}$ controls serotonin signaling in Caenorhabditis elegans. Genetics 178:157-169.

Trent C, Tsuing N, Horvitz HR (1983) Egg-laying defective mutants of the nematode Caenorhabditis elegans. Genetics 104:619-647.

Uvarov P, Ludwig A, Markkanen M, Pruunsild P, Kaila K, Delpire E, Timmusk T, Rivera C, Airaksinen MS (2007) A novel N-terminal isoform of the neuronspecific K-Cl cotransporter KCC2. J Biol Chem 282:30570-30576.

Waggoner LE, Zhou GT, Schafer RW, Schafer WR (1998) Control of alternative behavioral states by serotonin in Caenorhabditis elegans. Neuron 21:203-214.

Wake H, Watanabe M, Moorhouse AJ, Kanematsu T, Horibe S, Matsukawa N, Asai K, Ojika K, Hirata M, Nabekura J (2007) Early changes in KCC2 phosphorylation in response to neuronal stress result in functional downregulation. J Neurosci 27:1642-1650.

Woo NS, Lu J, England R, McClellan R, Dufour S, Mount DB, Deutch AY, Lovinger DM, Delpire E (2002) Hyperexcitability and epilepsy associated with disruption of the mouse neuronal-specific $\mathrm{K}-\mathrm{Cl}$ cotransporter gene. Hippocampus 12:258-268. 